\title{
EVOLUÇÃO AUDIOMÉTRICA EM USUÁRIOS DE IMPLANTE COCLEAR MULTICANAL
}

\section{Audiometric evolution in multichannel cochlear implant users}

\author{
Ana Cristina Hiromi Hoshino ${ }^{(1)}$, Dorilan Rodriguez da Cruz ${ }^{(2)}$, Maria Valéria Schmidt Goffi-Gomez ${ }^{(3)}$, \\ Débora Maria Befi-Lopes ${ }^{(4)}$, Carla Gentile Matas ${ }^{(5)}$, Talita M. Fortunato-Tavares ${ }^{(6)}$, Robinson Koji Tsuji ${ }^{(7)}$
}

\section{RESUMO}

Objetivo: analisar em quanto tempo após a ativação é possível alcançar os limiares auditivos desejados nos indivíduos usuários de implante coclear (IC) multicanal de diferentes faixas etárias. Método: estudo seccional retrospectivo a partir de banco de dados, aprovado pelo CEP/HCFMUSP, sob número 779/06. Foram incluídos indivíduos implantados no período de Janeiro de 2005(início da formação do banco de dados) a Setembro de 2008(término da coleta de dados), cujos prontuários possuíam dados dos limiares audiométricos pré-implante e 3, 6 e 12 meses após o IC. Setenta e dois pacientes contemplaram os critérios, divididos em dois grupos por faixa etária: GI (até 17 anos) e Gll (18 a 66 anos). Foi utilizada a Análise de Variância (ANOVA) de um fator para verificar a interação entre as médias dos limiares audiométricos e o tempo após o IC. Resultados: observou-se que 15 do grupo Gl já alcançaram limiares em torno de 30dBNA aos 3 meses pós implante. $O$ mesmo aconteceu com 26 pacientes do grupo Gll. Encontrou-se interação significante entre tempo $(F(3,140)=91,973, p=0,00)$ e a média de limiares auditivos. Nas análises Post-hoc do grupo Gl foram observadas diferenças estatisticamente significantes para as comparações entre AASI e 3 meses pós implante $(p=0,00)$ e entre 3 e 12 meses $(p=0,28)$, para o grupo Gll, somente para a comparação entre AASI e 3 meses $(p=0,00)$. Conclusão: uma mudança significante da audição pode ser observada a partir de três meses após o IC, porém este resultado não se encontra em todos os implantados.

DESCRITORES: Implante Coclear; Audiometria; Limiar Auditivo

\section{INTRODUÇÃO}

Os implantes cocleares tiveram avanços significantes nos últimos anos. Com isso, muitas crenças

(1) Fonoaudióloga; Pesquisadora colaboradora do Grupo de Implante Coclear da Faculdade de Medicina da Universidade de São Paulo HCFMUSP, São Paulo, Brasil; Doutoranda do Instituto de Estudos em Saúde Coletiva/Universidade Federal do Rio de Janeiro - IESC/UFRJ.

(2) Fonoaudiólogo; Ex-aluno do curso de Aprimoramento do Grupo de Implante Coclear da Faculdade de Medicina da Universidade de São Paulo HCFMUSP, São Paulo, Brasil.

(3) Fonoaudióloga da Divisão de Clínica ORL-HCFMUSP, coordenadora da equipe de Fonoaudiologia do Grupo de Implante Coclear da Faculdade de Medicina da Universidade de São Paulo - HCFMUSP, São Paulo, Brasil; Doutora em Ciências dos Distúrbios da Comunicação pela Universidade Federal de São Paulo -UNIFESP.

(4) Fonoaudióloga; Professora Associada do Departamento de Fonoaudiologia, Fisioterapia e Terapia Ocupacional da FMUSP, São Paulo, Brasil; Livre docente. se tornaram realidades e muitos fatos ficaram obsoletos. Estudar a evolução auditiva dos pacientes implantados é uma importante ferramenta para determinar o prognóstico dos pacientes, gerenciar

(5) Fonoaudióloga; Professora Associada do Departamento de Fonoaudiologia, Fisioterapia e Terapia Ocupacional da FMUSP, São Paulo, Brasil; Livre docente.

(6) Fonoaudióloga; Doutoranda em Ciências da reabilitação, Faculdade de Medicina da Universidade de São Paulo, São Paulo, Brasil; Ph.D. Candidate in Speech, Language, Hearing Sciences, The Graduate School, City University of New York.

(7) Médico Otorrinolaringologista; Professor Colaborador do Departamento de Otorrinolaringologia da FMUSP; Doutor em Medicina pela FMUSP.

Grupo de Implante Coclear da Faculdade de Medicina da Universidade de São Paulo - Departamento de Fisioterapia, Fonoaudiologia e Terapia Ocupacional

Conflito de interesses: inexistente 
as expectativas da família e reavaliar nossos critérios para candidatos ao implante coclear. As características de cada paciente na avaliação pré implante (tempo de surdez, uso de AASI, etiologia da doença e etc) são extremamente importantes para predizer o prognóstico deste 1 . A expectativa é que o usuário consiga não somente escutar, mas principalmente entender a linguagem oral. Porém, para o desenvolvimento das habilidades linguísticas acontecerem é necessário também o desenvolvimento das habilidades auditivas. Portanto, atenção e deteccção, habilidades primariamente adquiridas são pré requisitos para o desenvolvimento da comunicação ${ }^{2,3}$. A primeira habilidade auditiva que o implante coclear favorece é a detecção ${ }^{4}$. Todos os sons da fala precisam ser detectados pelo indivíduo para que a fala seja reconhecida e compreendida. Na Figura 1 observa-se a distribuição dos sons do português no audiograma. A figura mostra que alguns sons tem energia por volta de $40 \mathrm{~dB}$ em frequências baixas $(m, n)$ e outros tem energia por volta de $25 \mathrm{~dB}$ em frequências altas (s, f). Portanto, são necessários pelo menos $30 \mathrm{~dB}$ de limiar auditivo para ter acesso a todos os sons da fala, mesmo aqueles de fraca energia sonora ${ }^{5}$.

Freqüencia $(\mathrm{Hz})$

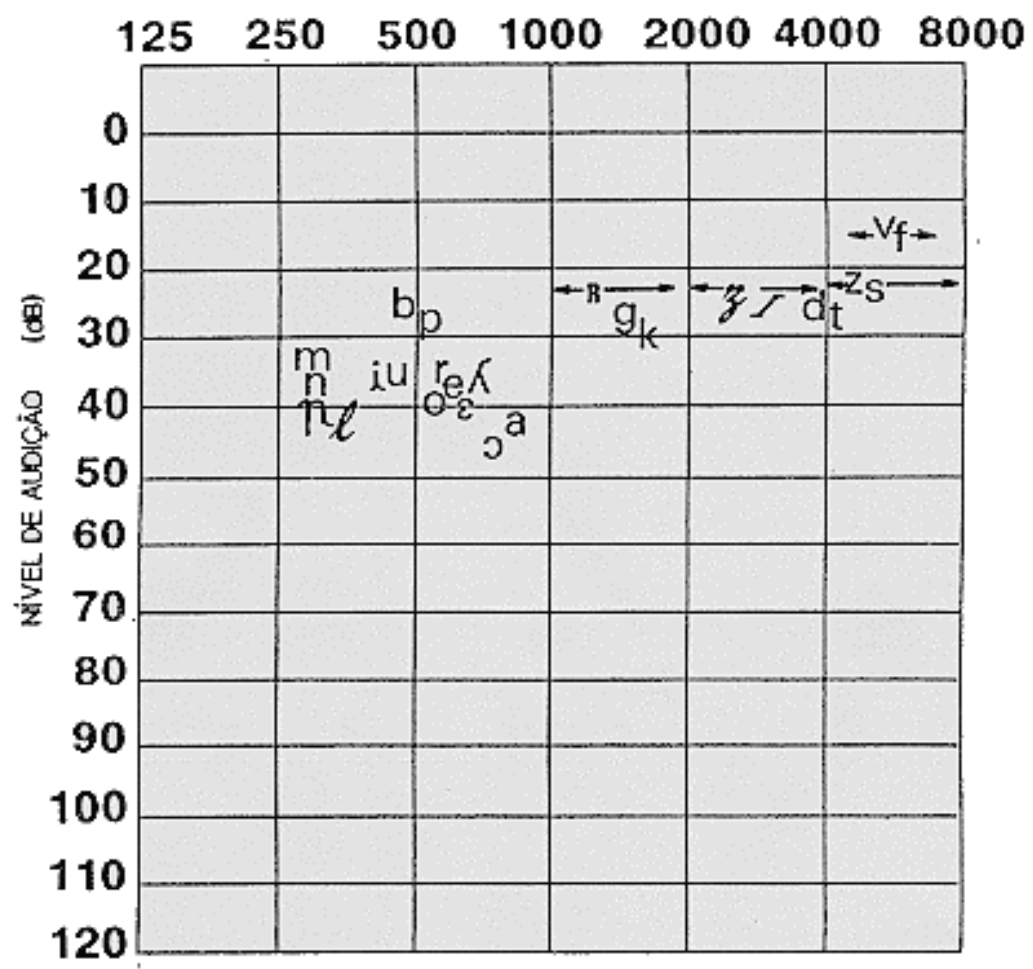

Figura 1 - Distribuição dos sons do português no audiograma, segundo Russo e Behlau (1993)

Com o uso do implante e o acúmulo de experiências auditivas a audição vai melhorando e um número maior de sons pode ser detectado. A confirmação dos níveis de detecção pode ser observada nas respostas obtidas em uma audiometria em campo sonoro.

Desta forma, a evolução das respostas audiométricas no primeiro ano de implante, se torna um importante instrumento de avaliação da evolução do paciente implantado.

Bento et al. (2004) ${ }^{6}$ avaliaram o resultado auditivo de 61 pacientes adultos implantados com o implante coclear multicanal por meio dos testes de reconhecimento de palavras e sentenças. A avaliação auditiva obtida seis meses após a primeira programação do implante demonstra que os pacientes de língua portuguesa obtêm excelentes resultados em testes de reconhecimento de palavras e sentenças em apresentação aberta, readquirindo uma audição útil.

Oyanguren et al. (2010) ${ }^{7}$ analisaram os benefícios do implante coclear nos idosos com relação à audibilidade, ao reconhecimento de fala e ao uso do telefone. Após um ano de uso de implante, a média dos limiares em campo passou a ser de 34 dBNA, a discriminação de frases em contexto aberto de 
$93,57 \%$ e $71 \%$ dos pacientes já conseguiam estabelecer uma conversa ao telefone. Os pacientes idosos usuários de implante coclear apresentaram ganhos auditivos relevantes, com melhora significante da compreensão em contexto aberto e no uso de telefones.

Samuel et al. (2010) ${ }^{8}$ verificaram a contribuição da atualização da tecnologia do IC sobre limiares audiométricos e desempenho na percepção de sentenças após 12 meses de uso do implante. Os usuários do Implante Coclear Nucleus 24 comparados aos usuários do Implante Coclear Nucleus N22 apresentaram melhores médias nos limiares audiométricos, mas os testes de percepção de sentenças no silêncio não indicaram diferença entre modelos. Os autores concluíram que a contribuição da tecnologia foi evidenciada apenas nos limiares audiométricos. Novos estudos estão sendo conduzidos para avaliar a contribuição da tecnologia na percepção de fala em situações auditivas mais difíceis.

O Objetivo do estudo foi analisar em quanto tempo após a ativação é possível alcançar os limiares auditivos desejados nos indivíduos usuários de IC multicanal de diferentes faixas etárias.

\section{MÉTODO}

É um estudo seccional retrospectivo que pesquisou o banco de dados do ambulatório do Grupo de Implante Coclear do Hospital das Clínicas da Faculdade de Medicina da Universidade de São Paulo, no período de Janeiro de 2005 (início da formação do banco de dados) a Setembro de 2008 (término da coleta de dados). Foram incluídos na amostra indivíduos com perda auditiva neurossensorial congênita ou adquirida, de grau severo a profundo bilateral, cujos prontuários possuíam dados dos limiares audiométricos com o uso do AASI na orelha implantada no pré-cirúrgico bem como os limiares audiométricos com 3, 6 e 12 meses de uso do IC. Setenta e dois prontuários de pacientes apresentavam os dados completos de acordo com os critérios da pesquisa. Estes foram divididos em dois grupos de 36: GI (um a 17 anos) e Gll (18 a 66 anos) para análise e comparação estatística.

O grupo GI, tinha idade média na data da cirurgia de 7,4 anos e média de 6,6 anos de privação auditiva. O grupo Gll, tinha idade média na data da cirurgia de 38,7 anos e média de 18 anos de privação auditiva. As etiologias mais comuns dos 72 sujeitos foram $50 \%$ desconhecidas, $10 \%$ traumática, $7 \%$ rubéola, $5 \%$ Sd Usher e $28 \%$ outras causas (Tabela 1).
Tabela 1 - Distribuição demográfica dos grupos GI e GII

\begin{tabular}{|c|c|c|}
\hline & GI & GII \\
\hline \multicolumn{3}{|l|}{ Idade } \\
\hline $0-5$ anos & 14 & \\
\hline $6-10$ anos & 16 & \\
\hline $11-19$ anos & 6 & \\
\hline 20-30 anos & & 9 \\
\hline $31-40$ anos & & 10 \\
\hline $41-50$ anos & & 9 \\
\hline 51-66 anos & & 8 \\
\hline \multicolumn{3}{|l|}{ Tempo privação } \\
\hline $0-5$ anos & 16 & 9 \\
\hline $6-10$ anos & 16 & 6 \\
\hline $11-20$ anos & 4 & 7 \\
\hline 21-30 anos & & 7 \\
\hline$>31$ anos & & 7 \\
\hline \multicolumn{3}{|l|}{ Etiologia } \\
\hline Desconhecida & 18 & 16 \\
\hline Rubéola & 4 & \\
\hline Ototoxicidade & 3 & \\
\hline Traumática & & 7 \\
\hline Otosclerose & & 3 \\
\hline S. Usher & 2 & \\
\hline Outras & 9 & 10 \\
\hline \multicolumn{3}{|l|}{ Tipo de IC } \\
\hline Nucleus 22 & & 1 \\
\hline Nucleus 24 M/K & 22 & 17 \\
\hline Nucleus Contour & 8 & 16 \\
\hline Nucleus Freedom & 2 & 1 \\
\hline Hires 90K & 3 & \\
\hline outros & 1 & 1 \\
\hline
\end{tabular}

Os 72 pacientes selecionados realizaram o ganho funcional do AASI bem como do implante coclear em cabina, audiômetro marca Madsen Midimate 622 conectado a uma caixa acústica por meio de um amplificador da acústica Orlandi. Estímulos sonoros do tipo warble foram apresentados em campo sonoro nas frequências de 500 a 8000 $\mathrm{Hz}$. Para a obtenção da resposta do limiar auditivo, foram usados os métodos convencionais, a audiometria de reforço visual e/ou a audiometria condicionada. Foi utilizada a média de $500 \mathrm{~Hz}$ a $4 \mathrm{kHz}^{9}$ para cálculos estatísticos.

Este trabalho foi aprovado pela Comissão de Ética da Instituição (CAPPesq HCFMUSP), sob processo número 779/06. Todos os participantes e/ou responsáveis assinaram o Termo de Consentimento Livre e Esclarecido, conforme Resolução 
196/96 (BRASIL Resolução MS/CNS/CNEP no 196/96 de 10 de outubro de 1996).

A análise estatística foi realizada no programa SPSS. Foram usados os testes de Análise de variância (ANOVA) de um fator (tempo) para verificar a interação das médias dos limiares audiométricos e de Repeated Measures ANOVA para análise dos valores médios dos limiares entre grupos.

\section{RESULTADOS}

A Tabela 2, e as Figuras 2 e 3 mostram que tanto as crianças (Grupo I) como os adultos (Grupo II) tem grande melhora nos limiares auditivos três meses após o implante coclear. Entretanto, o acesso aos sons de fala pode demorar a ser alcançado. Alguns indivíduos não alcançam limiares melhores do que $30 \mathrm{~dB}$ mesmo após 12 meses do implante.

Tabela 2 - Dados da estatística descritiva das médias dos limiares audiométricos (dBNA) das crianças (Grupo I) e dos indivíduos adultos (Grupo II)

\begin{tabular}{lcccccc}
\hline & \multicolumn{3}{c}{ Grupo I } & \multicolumn{3}{c}{ Grupo II } \\
\cline { 2 - 7 } & Média & Mínimo & Máximo & Média & Mínimo & Máximo \\
\hline Pré Implante (AASI) & 103.73 & 60.83 & 130.00 & 102.22 & 55.00 & 130.00 \\
Pós 3 meses de IC & 52.03 & 19.17 & 130.00 & 39.12 & 16.67 & 80.00 \\
Pós 6 meses de IC & 42.22 & 19.17 & 95.00 & 37.79 & 13.33 & 94.17 \\
Pós 12 meses de IC & 40.02 & 24.17 & 83.33 & 34.98 & 15.83 & 82.50 \\
\hline
\end{tabular}

Média dos limiares audiométricos em campo sonoro das crianças, antes do IC com AASI e após o implante coclear

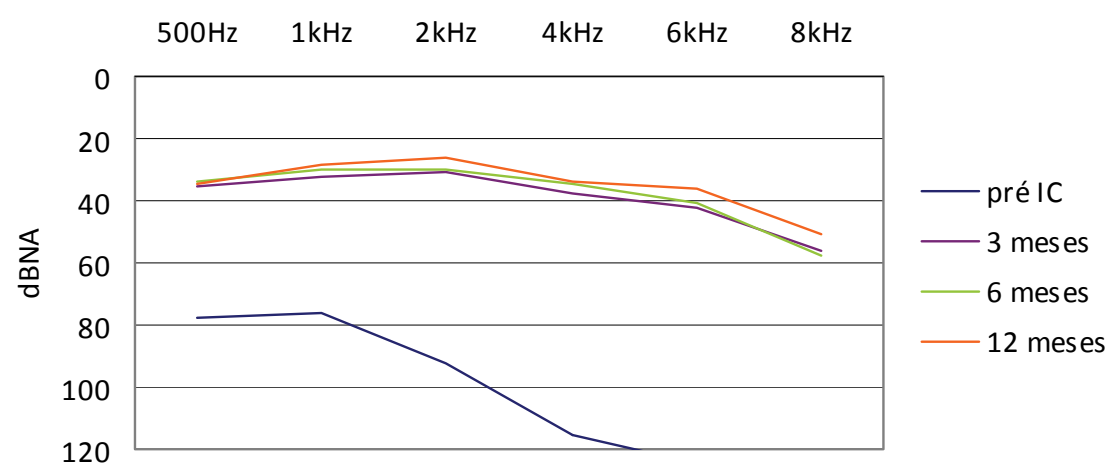

Figura 2 - Média dos limiares audiométricos para o grupo GI

Média dos limiares audiométricos em campo sonoro dos adultos, antes do IC com AASI e após o implante coclear

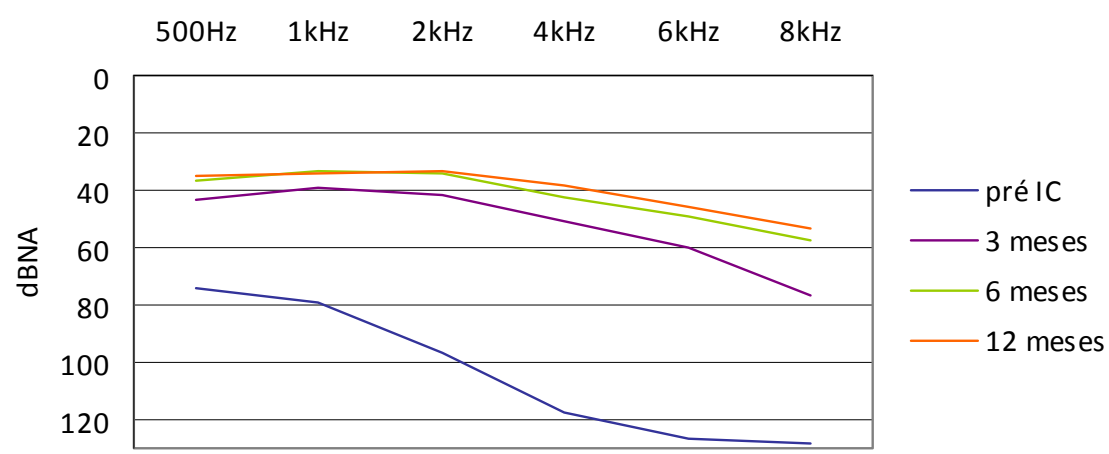

Figura 3 - Média dos limiares audiométricos para o grupo Gll 
$\mathrm{Na}$ análise das médias dos limiares audiométricos obtidos aos 3 meses pós implante dos pacientes implantados,observa-se que 15 pacientes do grupo Gl já alcançaram limiares em torno de 30dBNA. O mesmo aconteceu com 26 pacientes do grupo Gll (Tabela 3).

Tabela 3 - Distribuição dos indivíduos e as respectivas médias dos limiares audiométricos alcançadas na avaliação auditiva após 3 meses de uso do Implante Coclear

\begin{tabular}{ccccccc}
\hline & Até 30dBNA & 31 a 40dBNA & 41 a 70dBNA & 71 a 90dBNA & >91dBNA & Total \\
\hline Gl & 15 & 11 & 8 & 2 & 0 & 36 \\
Gll & 26 & 5 & 5 & 0 & 0 & 36 \\
\hline
\end{tabular}

A análise de Variância (ANOVA) de um fator (tempo) foi utilizada para verificar a interação entre as médias dos limiares de $500 \mathrm{~Hz}, 1 \mathrm{kHz}, 2 \mathrm{kHz}$ e $4 \mathrm{kHz}$, obtidos em campo sonoro antes (AASI) e 3, 6 e 12 meses após a cirurgia (IC) para ambos os grupos GI e Gll. Com relação ao grupo GI, foi verificada uma interação significante $(F(3,140)=91,973$, $p=0,00$ ) entre tempo e média de limiares auditivos (Figura 4). Análises Post-hoc foram utilizadas para verificar mais precisamente esta interação por meio de quatro adicionais ANOVAs (Tabela 4). Foi observada diferença estatisticamente significante para as comparações entre AASI e 3 meses pós implante $(p=0,00)$ e entre 3 e 12 meses pós implante $(p=0,28)$. Observou-se também uma tendência à significância estatística na comparação da média dos limiares entre 3 e 6 meses pós implante $(p=0,06)$.

Análise Intergrupos

$F(4,67)=2.2297, p=.07501$

Barras verticais denotam IC de 0,95

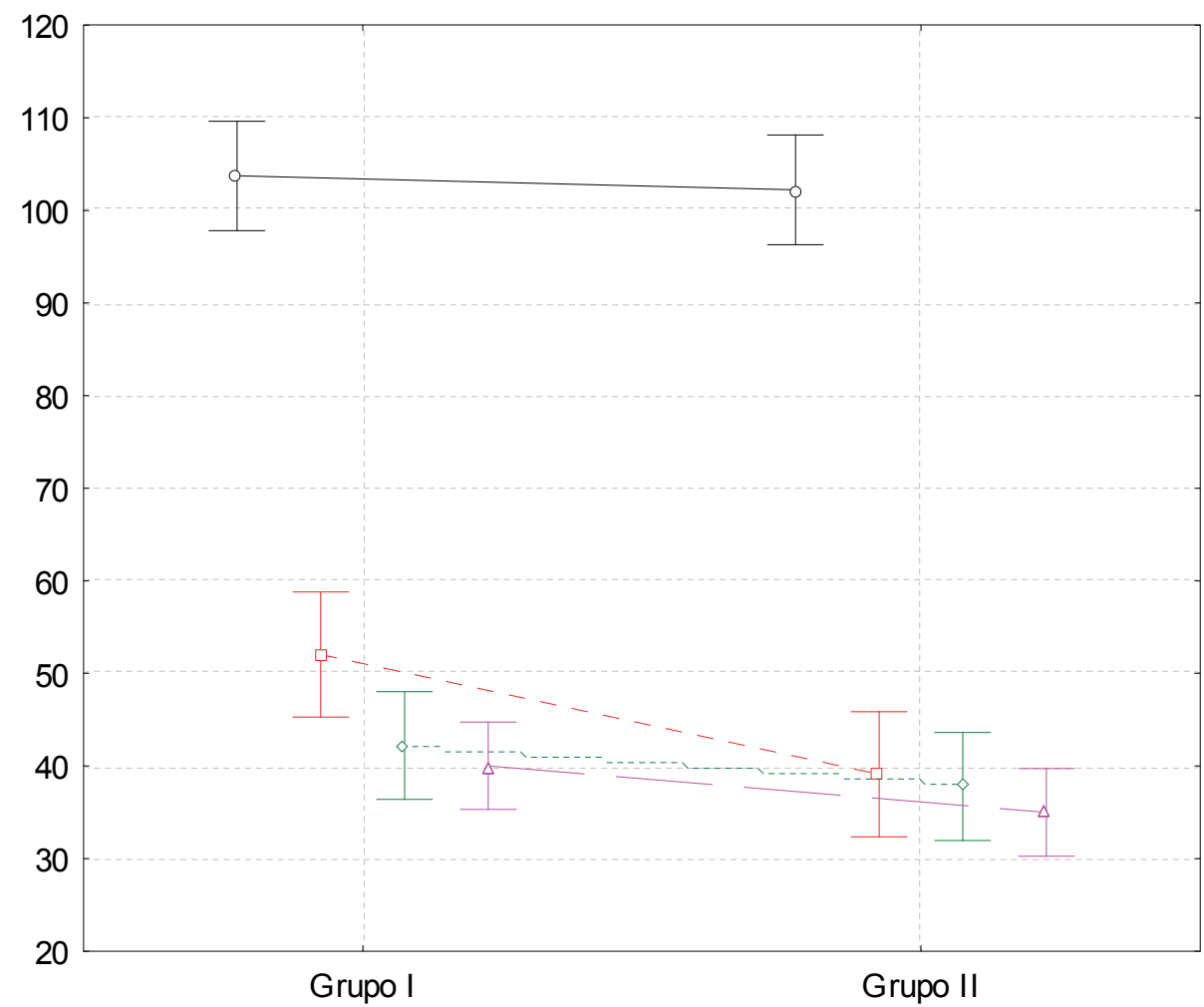

Pré IC (AASI)

Pós 3 meses IC Pós 6 meses IC Pós 12 meses IC

Figura 4 - Comparação intergrupos relativa às medidas dos limiares Pré IC (AASI) e 3,6 e 12 meses Pós IC 
Tabela 4 - Análises POST-HOC para grupo I (GI)

\begin{tabular}{cccccc}
\hline & SS & Df & MS & F & P \\
\hline AASI vs 3 IC & 48100 & 1 & 48100 & 107.60 & $.000^{*}$ \\
3 IC vs 6 IC & 1732.00 & 1 & 1732.00 & 3.81 & 0.06 \\
6 IC vs 12 IC & 48.62 & 1 & 48.62 & 0.19 & 0.67 \\
3 IC vs 12 IC & 2951.00 & 2 & 1475.00 & 3.77 & $.028^{*}$ \\
\hline
\end{tabular}

ANOVAs de um fator (significância estatística considerada a $95 \%-\mathrm{p}^{*}$ denotam valores estatisticamente significantes).

Para o grupo Gll, foi constatada uma interação significante $(F(3,140)=91,973, p=0,00)$ entre tempo e média de limiares auditivos (Figura 4). Nas análises Post-hoc (Tabela 5), foi observada diferença estatisticamente significante somente para a comparação entre AASI e 3 meses pós implante $(p=0,00)$.

Tabela 5 - Análises POST-HOC para grupo II

\begin{tabular}{cccccc}
\hline & SS & df & MS & F & P \\
\hline AASI vs 3 IC & 71700 & 1 & 71700 & 254.50 & $0.00^{*}$ \\
3 IC vs 6 IC & 32 & 1 & 32 & 0.12 & 0.73 \\
6 IC vs 12 IC & 142.10 & 1 & 142.10 & 0.56 & 0.46 \\
3 IC vs 12 IC & 309 & 1 & 309 & 1.35 & 0.25 \\
\hline
\end{tabular}

ANOVAs de um fator (significância estatística considerada a 95\%0 - p* denota valore estatisticamente significante).

Para analisar a interação dos valores das médias de limiares entre os grupos GI e GII (Figura 4 ), realizou se Repeated Measures ANOVA que não foi estatisticamente significante $(p=0,075)$.
Análises Post-hoc por meio de ANOVAs de um fator (Tabela 6) demonstraram que houve diferença intergrupos estatisticamente significante aos 3 meses pós implante $(p=0,009)$.

Tabela 6 - Análises POST-HOC para a comparação intergrupos GI e GII

\begin{tabular}{lccccc}
\hline & SS & Df & MS & F & P \\
\hline Pré IC (AASI) & 40.75 & 1 & 40.75 & 0.129 & 0.721 \\
Pós 3 meses IC & 3001 & 1 & 3001 & 7.277 & $.009^{*}$ \\
Pós 6 meses IC & 354.1 & 1 & 354.1 & 1.157 & 0.286 \\
Pós 12 meses IC & 458.4 & 1 & 458.4 & 2.258 & 0.137 \\
\hline
\end{tabular}

ANOVAs de um fator (significância estatística considerada a $95 \% 0-p^{*}$ denota valor estatisticamente significante)

\section{DISCUSSÃO}

Comparando os limiares auditivos pré IC (AASI) e após três meses de uso de IC é possível observar uma diferença estatisticamente significante tanto para o grupo GI quanto para o GII $\left(p=0,00^{*}\right)$. Além de confirmar o maior benefício propiciado pelo IC em comparação ao AASI, fornece evidência de que uma diferença significante do limiar auditivo já pode ser observada a partir dos três meses após a cirurgia. Como foi dito anteriormente, ressalta-se que os resultados da percepção auditiva e de fala de um paciente implantado são variáveis. De acordo com sua história pregressa, seu desempenho pode variar no tempo e na qualidade auditiva ${ }^{10}$.

Apesar de não haver uma relação estatísticamente significante no grupo GI para comparação de 3/6meses e 6/12 meses de uso do IC, observa-se 
uma evolução contínua dos limiares por meio da relação $3 / 12$ meses $(p=0.028)$. Tais comparações nos levam à compreensão dos benefícios propiciados pelo IC em crianças implantadas em diversas idades, amplamente discutidos na literatura ${ }^{11,12}$. Efetivamente o implante coclear permite a detecção de sons de fraca energia, favorecendo o desenvolvimento das demais habilidades auditivas.

Holt e Svirsky (2008) ${ }^{13}$ descrevem em sua pesquisa que o desenvolvimento progressivo da linguagem oral é mais rápido em crianças implantadas precocemente comparadas àquelas implantadas depois dos quatro anos de idade, porém, o score de percepção de fala em contextos abertos é similar, sugerindo assim que o desenvolvimento do reconhecimento da palavra parece ser similar para crianças implantadas de 1 até 4 ano de idade.

Em contrapartida, no grupo GII, apesar das médias dos limiares audiométricos mostrarem a mesma evolução do grupo GI, não foi possível estabelecer uma relação estatísticamente significante para comparação de 3/6, 6/12 e 3/12 meses.

Budenz et al. (2011) ${ }^{14}$ acreditam que adultos em idade avançada, pós linguais, implantados, obtêm um grande benefício na percepção de fala. Obviamente os scores não são parecidos com a de adultos jovens. Os autores acreditam também que as diferenças de desempenho estão correlacionadas com a duração da perda auditiva.
Na análise intergrupos GI e GII, pode-se concluir que ambos apresentaram diferenças estatisticamente significantes para o período de 3 meses, demonstrando assim que os pacientes, independentemente da idade que foi implantado, tempo de privação sensorial e desempenho das habilidades auditivas e linguísticas pré implante desenvolvem novas habilidades logo no início da ativação. As mudanças auditivas afetam a percepção da fala e quanto mais rápido for sua adaptação, melhor será sua comunicação oral. A adaptação é um processo no qual o indivíduo adquire novos conhecimentos e modifica a sua habilidade ${ }^{15}$.

Os resultados mostram que a detecção de sons de média e fraca intensidade é possível para a maioria das pessoas implantadas, adultos e crianças, entretanto, algumas pessoas demoram mais tempo para alcançar o acesso aos sons de fala.

\section{CONCLUSÃO}

Uma diferença significante no nível de audição já pode ser observada a partir de três meses após a cirurgia de IC, tanto em adultos como em crianças, porém este resultado não é preditivo para todos os implantados.

\begin{abstract}
Purpose: to evaluate how long after activation one can achieve the desired auditory thresholds in individuals multichannel cochlear implant users from different age groups. Method: a retrospective cross-sectional from a database, approved by CEP / FMUSP, under number 779/06. Individuals implanted between January 2005 and September 2008, whose files had preoperative audiometric data and 3, 6 and 12 months after $\mathrm{Cl}$ were included. Seventy-two patients fulfilled the criteria, and they were divided in two groups according to age: group I (17 year old) and GII (18-66 year old). Analysis of variance (ANOVA) of one factor in order to verify the interaction between the mean threshold and time after $\mathrm{Cl}$ were used. Results: we observed that 15 of the $\mathrm{Gl}$ have reached around 30dBNA thresholds at 3 months after implant. The same happened to 26 patients of Gll. We found significant interaction between time $(F(3.140)=91.973, p=0.00)$ and the average hearing thresholds. Post-hoc analysis in the GI group were statistically significant differences for comparisons between $\mathrm{HA}$ and 3 months after implantation $(p=0.00)$ and between 3 and 12 months $(p=0.28)$. For Gll only for comparison between $\mathrm{HA}$ and 3 months $(p=0.00)$. Conclusion: a significant hearing change may be observed beginning from three months after IC, but this result is not found in all implanted subjects.
\end{abstract}

KEYWORDS: Cochlear Implantation; Audiometry; Auditory Threshold 


\section{REFERÊNCIAS}

1. Santarelli R, Magnavita V, De Filippi R, Ventura L, Genovese E, Arslan E. Comparison of speech perception performance between Sprint/Esprit 3G and Freedom processors in children implanted with nucleus cochlear implants. Otol Neurotol. 2009;30(3):304-12.

2. Pereira LD \& Schochat E. Processamento Auditivo Central: Manual de avaliação. $1^{\text {a }}$ ed. São Paulo, LOVISE. p.99-102, 1997.

3. Muniz LF, Roazzi A, Schochat E, Teixeira CF, Lucena JA. Avaliação da habilidade de resolução temporal, com uso do tom puro, em crianças com e sem desvio fonológico. Rev. CEFAC. 2007;9 (4): 550-62.

4. Engelmann L, da Costa Ferreira MID. Avaliação do processamento auditivo em crianças com dificuldades de aprendizagem. "Auditory processing evaluation in children with learning difficulties". Rev Soc Bras Fonoaudiol. 2009;14(1):69-74.

5. Russo ICP, Behlau MS. Percepção da fala: análise acústica do português brasileiro. São Paulo, Lovise. P.57,1993.

6. Bento RF, de Brito Neto R, Castilho AM, Gómez VG, Giorgi SB, Guedes MC. Resultados auditivos com o implante coclear multicanal em pacientes submetidos a cirurgia no Hospital das Clínicas da Faculdade de Medicina da Universidade de São Paulo. Rev Bras Otorrinolaringol. 2004; 70 (5): 632-7.

7. Oyanguren V, Gomes MVSG, Tsuji RK, Bento RF, de Brito Neto RV. Resultados audiológicos do implante coclear em idosos. Braz J Otorhinolaryngol. 2010;76(4):450-3.
8. Samuel PA, Goffi-Gomez MVS, Lopes DMB, Matas CG, Tsuji RK, de Brito Neto RV, Bento RF. Percepção de Fala e Limiares Audiométricos em Usuários de Implante Coclear Nucleus 22 e Nucleus 24. Arq. Int. Otorrinolaringol, São Paulo - Brasil. Jul/ Ago/Setembro, 2010;14(3): 331-7.

9. BIAP- Bureau International d'AudioPhonologie, 1996. Disponível em: www.biap.org/biapanglais/ rec021eng.htm.

10. Green KMJ, Bhatt YM, Mawman DJ, O'Driscoll MP, Saeed SR, Ramsden RT, Green MW. Predictors of audiological outcome following cochlear implantation in adults Cochlear Implants International. Cochlear Implants Int. 2007; 8(1): 1-11. Published online in Wiley InterScience.

11. Tajudeen BA, Waltzman SB, Jethanamest D, Svirsky DA. Speech Perception in Congenitally Deaf Children Receiving Cochlear Implants in the First Year of Life Otology \& Neurotology 2010;31:1254-60.

12. Colletti L, Mandala M, Zoccante L, Shannon RV, Colletti V. Infants versus older children fitted with cochlear implants: Performance over 10 years. Int $\mathrm{J}$ Pediatr Otorhinolaryngol. 2011 Jan 28; 75(4):504-9. 13. Holt RF, Svirsky MA. An exploratory look at pediatric cochlear implantation: is earliest always best? Ear Hear. 2008;29(4):492-511.

14. Budenz CL, Cosetti MK, Coelho DH, Birenbaum B, Babb J, Waltzman SB, Roehm PC. The effects of cochlear implantation on speech perception in older adults. J Am Geriatr Soc. 2011 Mar;59(3):446-53.

15. Boothroyd A. Adapting to changed hearing: the potential role of formal training. J Am Acad Audiol. 2010 Oct;21(9):601-11.
http://dx.doi.org/10.1590/S1516-18462012005000037

RECEBIDO EM: 07/07/2011

ACEITO EM: 20/10/2011

Endereço para correspondência:

Ana Cristina Hiromi Hoshino

R. Capote Valente, 432 - cj 14

São Paulo - SP

CEP: 05409-001

E-mail: choshino@uol.com.br 